\title{
Can conductive adhesives with nanoparticles beat commonly used electrically conductive adhesives without nanoparticles?
}

\author{
David Bušek, Radoslav Radev, Pavel Mach \\ Czech Technical University in Prague \\ Department of Electrotechnology, Faculty of Electrical Engineering, \\ Technická 2, 16627 Prague 6, Czech Republic \\ E-mail: busekd1@fel.cvut.cz,rado.r@centrum.cz, mach@fel.cvut.cz
}

\begin{abstract}
This paper deals with electrically conductive adhesives (ECA's) with isotropic properties (ICA's) and describes the way of improving the quality parameters of such electrically conductive joint. This paper discusses the advantages and disadvantages of nanoparticle addition into commonly fabricated electrically conductive adhesive. Electrical parameters of ICA joints before and after mechanical, humid and humid-heat stress is applied, are observed. Standard controlling and evaluating principles for ECAs are based on resistance measurement of a joint; this paper shows that nonlinearity evaluation is more sensitive. For some applications, linearity might have crucial importance and an absolute size of resistance might not be so crucial. Our previous work proves that nonlinearity might not just be more sensitive but that it also does not have to progress in the same way as the resistivity [1].
\end{abstract}

\section{INTRODUCTION}

Electrical parameters of the electrical connection between the substrate and the attached component play a key role in quality and reliability of the whole electronic device. RoHS directive for the Europe Union took effect on July 1, 2006, and since this directive restricts (among other five hazardous materials) the use of lead in the manufacture of various types of electronic and electrical equipment, other ways to create a stable and reliable electrical joint are searched. Possible replacements are lead free solders and Electrically Conductive Adhesives (ECA's). Adhesives have many unsubstituable properties. Their main advantage is low curing temperature that can even be $20^{\circ} \mathrm{C}$. Mainly $150{ }^{\circ} \mathrm{C}$ are used to speed up the curing process. When this temperature is compared to the soldering lead-free techniques where the needed temperature varies around $250{ }^{\circ} \mathrm{C}$ or more, this advantage is obvious. Thermally sensitive devices can be attached without any harm. LCD's for example can only be attached using low temperature technologies. Their disadvantage at this time is higher price, lower time stability and also electrical parameters of electrically conductive joints that are up to now significantly worse than those with lead- or lead-free solders [1]. This is not a big problem for low current applications and LCDs, but some electronic devices might not work correctly if a joint with higher resistance or nonlinearity is present. One of the ways to reduce the resistance of a connection and its nonlinearity is, according to our measurements, to add conductive nanoparticles (balls or flakes) of a specified size into commonly fabricated ECAs. Standard measurement observe only the change of the resistance, in our measurement, nonlinearity which is more sensitive was also used. The principle was published in [2]. Our previous measurements were focused on conductive adhesives without nanoparticles, their comparison, advantages and disadvantages and usability in different conditions. One of these previously examined adhesives was selected for this experiment as a basic material, where the nanoparticles were added. 


\section{TYPES OF ELECTRICALLY CONDUCTIVE ADHESIVES USED IN THE EXPERIMENT}

Our experiment was grounded on one type of ECA that was chosen from tests made within past years. This adhesive (AX20 from Amepox company), that was selected for this experiment as a basic material, was enriched with nanoparticles. Two different types of nanoparticles and two different concentrations of nanoparticles were used. The specifications of the adjusted adhesives are in the table 1 below.

\begin{tabular}{|l|l|}
\hline $\begin{array}{l}\text { Type: Amepox AX 20 } \\
\text { (ECO Solder) }\end{array}$ & $\begin{array}{l}\text { Composition: } \\
\text { bisphenol epoxy resin } \\
+ \text { formulated } \mathrm{BF}_{3} \\
75 \% \mathrm{Ag} \text { flakes }\end{array}$ \\
\hline Amepox AX20 S1 & AX20+nanoparticles \\
& $80-100 \mathrm{~nm}$, weight \\
& percentage: $\mathbf{3 , 8} \%$ \\
\hline Amepox AX20 S2 & AX20+nanoparticles \\
& $\mathbf{6}-\mathbf{8 n m}$, weight \\
& percentage: $\mathbf{3 , 8} \%$ \\
\hline Amepox AX20 S3 & AX20+nanoparticles \\
& $80-$ 100nm, weight \\
& percentage: $\mathbf{7 , 4} \%$ \\
\hline Amepox AX20 S4 & AX20+nanoparticles \\
& $\mathbf{6}-\mathbf{8 n m}$, weight \\
& percentage: $\mathbf{7 , 4} \%$ \\
\hline
\end{tabular}

Tab. 1. Tested electrically conductive adhesives.

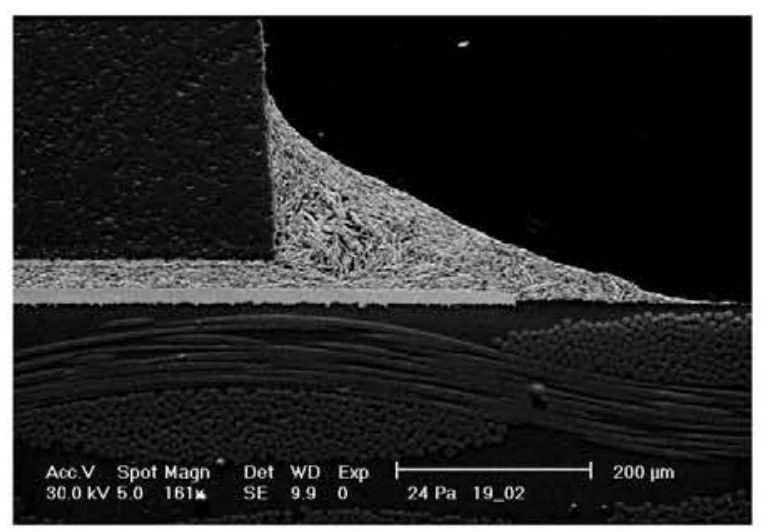

Fig. 1. Cross-Section of a tested conductive joint

\section{Preparation of Tested Samples aND APPLIED STRESS}

Printed circuit board samples (see Fig.2) were assembled with seven resistors of zero nominal resistance using electrically conductive adhesives. Dispense deposition was used and all adhesives were cured using $150^{\circ} \mathrm{C}$ in an oven with a flat temperature profile.

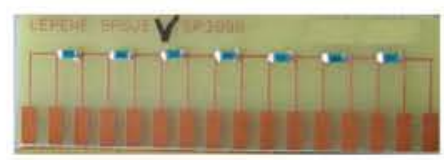

Fig. 2. Tested sample.

On these samples a series of measurement was carried out. Electrical parameters were observed. These were resistance (using four-point method) and nonlinearity (using method based on intermodulation signals, see fig.5).

\subsection{Mechanical stress}

Samples were exposed to cyclic mechanic bending where deflection was $\pm 4,5$ (see principle on fig.3). In the first step, the samples were exposed to 1000 cycles with the frequency of $1 \mathrm{~Hz}$. After remeasurement, these samples were exposed to another 1000 cycles and were once again re-measured.

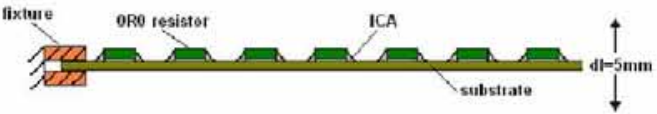

Fig. 3. Realization of cyclic mechanical stress. The sample consists of seven ORO resistors attached to an FR4 substrate.

\subsection{Climatic stress}

Samples were exposed to humid ambient in a closed container. Both types of stress lasted 100 hours. Humid stress was done in $95 \%$ RH and humidheat stress was done in $85^{\circ} \mathrm{C}$ and $100 \% \mathrm{RH}$. The observed electrical parameters were then measured and results discussed. Experimental results are shown in figures in part 5 - Experimental results. 


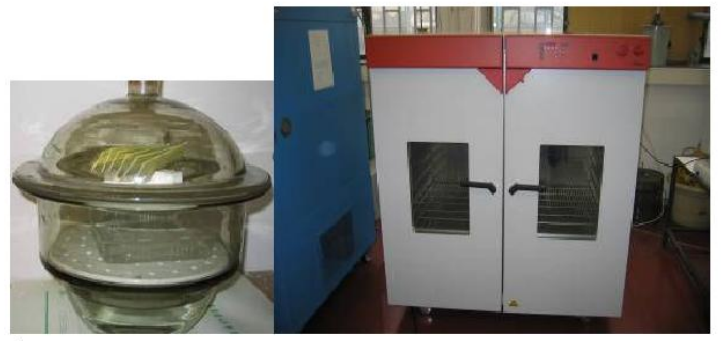

Fig. 4. Realization of humid and humid-heat stress. Samples were closed in an airtight container above the water level. This container was placed in the oven

\section{Methods of Measurement}

\subsection{Electrical resistance}

Electrical resistance was measured using fourpoint method; resistance of one joint can be calculated via the following equation:

$$
R_{\text {joint }}=\frac{R_{M}-R_{0}}{2}
$$

Where:

$\mathrm{R}_{\mathrm{M}} \ldots$ measured value

$\mathrm{R}_{0} \ldots 17 \mathrm{~m} \Omega(17 \pm 1) \mathrm{m} \Omega$ (discovered experimentally)

\subsection{Nonlinearity measurement}

Nonlinearity was examined by the use of two frequencies that were brought to the sample and the out-coming intermodulation frequency was examined (see principle in fig.5.). The higher the level of this intermodulation frequency, the higher the nonlinearity was.

\section{EXPERIMENTAL RESULTS}

Experimental results gained using different adhesive types and different applied stresses are illustrated in graphs below.

After mechanical stress was applied, electrical parameters of all adhesives worsened as expected, the biggest change occurred in sample 0 (S0) where no nanoparticles were added, best results were obtained for samples S1 and S3 where nanoparticles with approximate size $100 \mathrm{~nm}$ were present (see fig.6 and fig.7). The amount of added nanoparticles didn't play significant role, there is no further improvement for S3 where the double amount of nanoparticles is present. S3 even shows slightly worse parameters then $\mathrm{S} 1$. The possible explanation might be the same as for S2 and S4. Either there was a problem during preparation of the adhesive in the external company or the surface of the particles was too large and as they were covered with the epoxy, there was insufficient amount of the adhesive for proper mechanical function of the conductive mixture. Under standard curing conditions, the adhesive shrinks and therefore causes the particles to come closer to each other and improve overall conductivity. Another possible explanation for bad results with $\mathrm{S} 2$ and $\mathrm{S} 4$ is that smaller silver particles are more likely to oxidize faster and therefore they became an insulating layer. Samples S2 and S4 had significantly higher viscosity and therefore they needed to be thinned down with appropriate solvent. It is possible that too much solvent was necessary and the structure was irreversibly crippled.

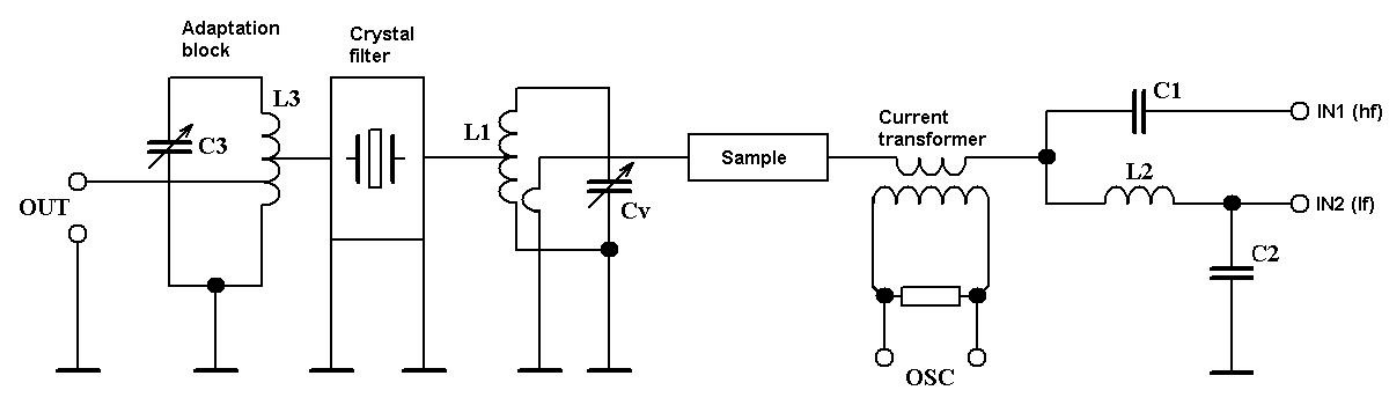

Fig.5. Circuit diagram of nonlinearity measurement 


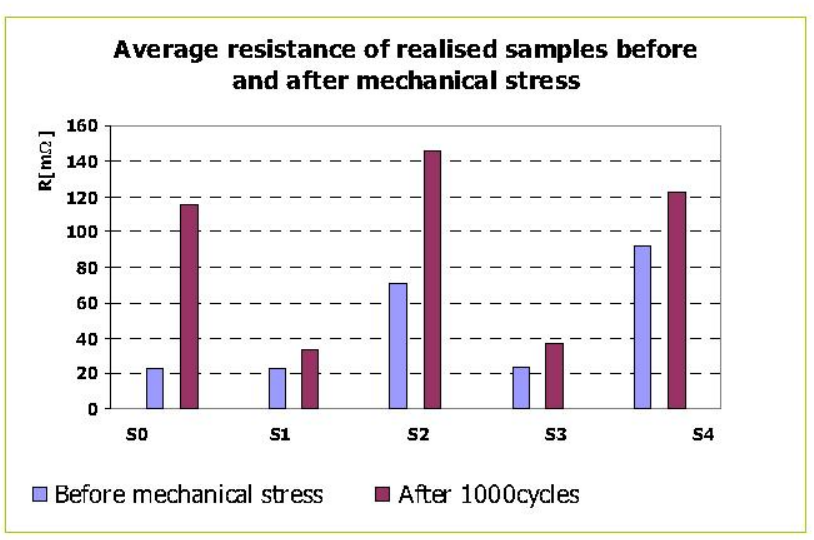

Fig. 6. Mechanical stress does not influence samples $S 1$ and S3 much

The graph below (fig.7) illustrates the same situation as in fig. 6 but this time, nonlinearity is presented. The difference after stress is applied; and differences among the adhesives themselves are so vast that logarithmic scale must be used. This proves that nonlinearity measurement is far more sensitive than resistivity measurement. $\mathrm{S} 1$ in terms of resistivity shows $43 \%$ change meanwhile the same sample in terms of nonlinearity shows nearly $3000 \%$ change. Should the mechanical stress be lower, measuring the resistance (in contrast to nonlinearity) would not show any change.

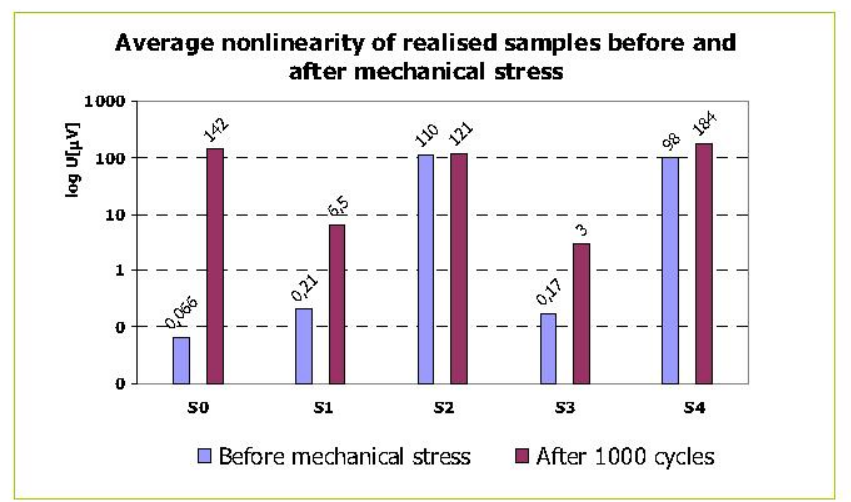

Fig. 7. Impact of mechanical stress in terms of nonlinearity ( $\log$ scale must be used)

Resistance measurement is not suitable for evaluating very small changes though; but has contrary wise other advantages. If the resistance is already high, the resistance after stress gets even higher, while nonlinearity stagnates and shows only a little increase (see fig. 8 representing $\mathrm{S} 2$ after 1000 and 2000 cycles). The results for 2000 cycles are not depicted in the graphs, as they worsened the parameters of all samples too much.

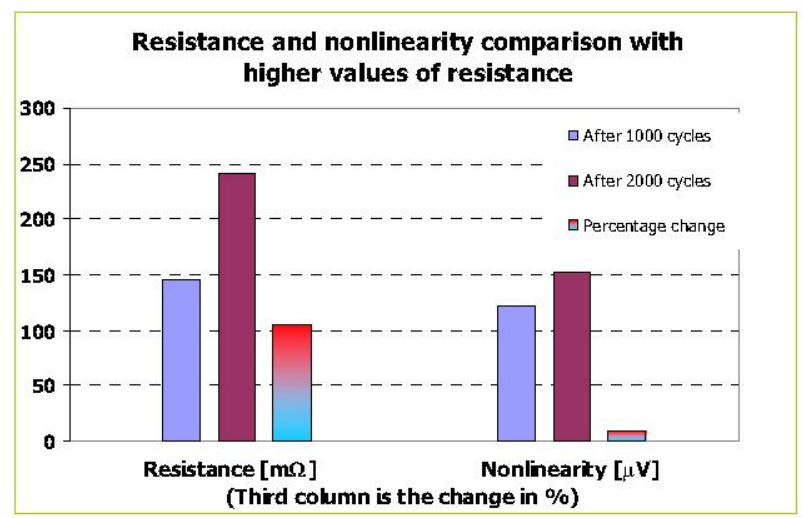

Fig. 8. Disadvantage of nonlinearity measurement exists for higher initial values of resistance

The immunity against atmospheric humidity was also tested. The samples were exposed for 100 hours and after the test, they were kept in standard laboratory conditions for one day before the measurement was conducted. Samples S2 and S4 showed in all climatic tests (fig.9 and later) their absolute inability to resist moisture. Therefore I will not comment on them about these tests. Generally, moisture within standard laboratory temperature range caused only little impact when compared to humid-heat test $\left(85^{\circ} \mathrm{C}, 100 \% \mathrm{RH}\right)$. The percentage change after humid test was about $20 \%$ for S1 and S3 (see fig.8) while unmodified S0 changed the resistance only $2 \%$. Nanoparticles did not improve the immunity against humidity in any way.

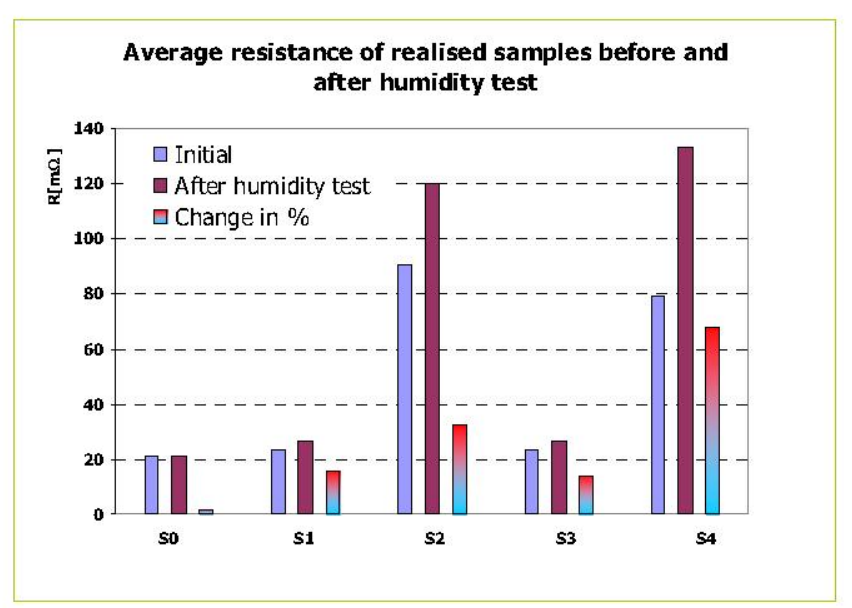

Fig. 8. Impact of humid stress on resistance

Even though resistance was almost without any interesting change, nonlinearity showed change from $9 \%(\mathrm{~S} 0)$ to $130 \%(\mathrm{~S} 1)$. Please note that the "y" axis has logarithmic scale (see fig.9). 


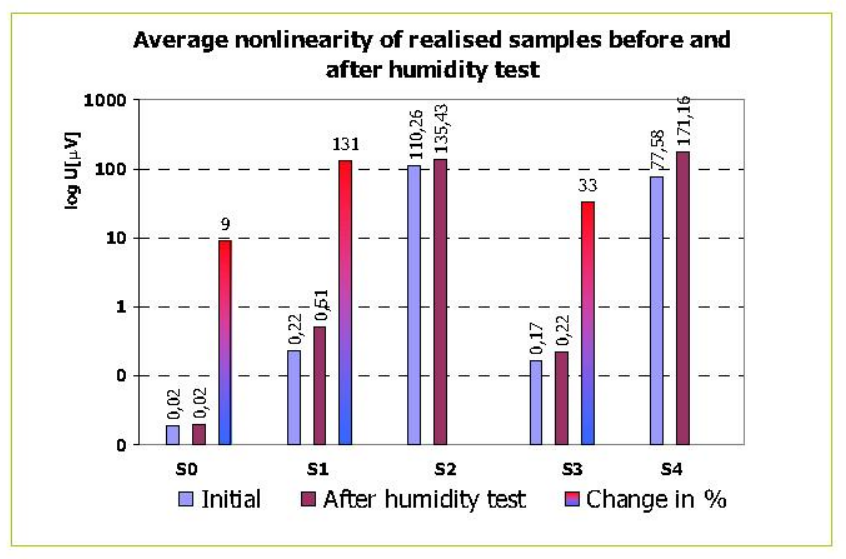

Fig. 9. Impact of humid stress on nonlinearity

Humid-heat stress had greatest impact on modified adhesive joints ( $\mathrm{S} 1$ to $\mathrm{S} 4$ ), the nonlinearity percentage change was $147 \%$ for S3, and $15000 \%$ for S1 (once again, logarithmic scale is used for better clearance), unmodified S0 sample resistance change was only $5 \%$ and nonlinearity change was only $35 \%$.

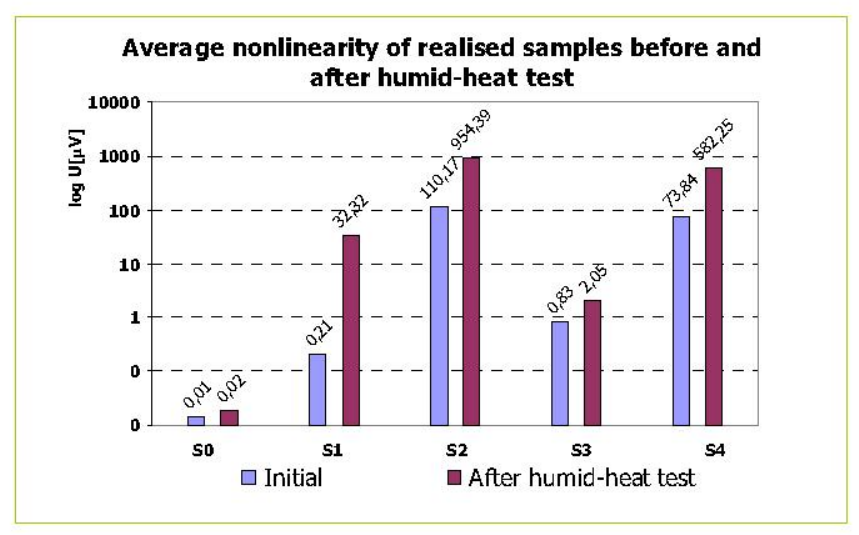

Fig. 11. Impact of humid-heat stress on nonlinearity

\section{CONClusion}

Altogether, five different adhesives were used. S0 was unmodified ECA "Eco Solder AX20" from Amepox company and other S1, S2, S3 and S4 were based on S0 but with the addition of different nanoparticles in different amounts (see part 2 above for details). S2 and S4 didn't show good parameters and therefore I will comment more on the others. S1 and $\mathrm{S} 3$ showed significant improvement in resistance against mechanical stress (see fig.6). Measuring nonlinearity instead of ohmic resistance brings higher sensitivity, but if the resistance already has high values, then the nonlinearity stagnates and keeps already high level (the change is minimal, see fig. 8 ).
Observing the electrical parameters after humid stress and humid-heat stress showed that addition of nanoparticles literally ruins the good resistance of unmodified EcoSolder AX20 against humidity. Therefore, it is not easy to answer the question in the topic of this paper: Can conductive adhesives with nanoparticles beat commonly used electrically conductive adhesives without nanoparticles? Not yet. In humidity free environments where mechanic stability is a plus definitely yes (if the concentration and proper size of nanoparticles is selected), where higher humidity is present definitely no. Further search must be conducted and some other improvements must be made before nanoparticles can be a real advantage.

This research was supported by grant: Czech Republic - MSM No.6840770021 - Diagnostic of Materials

\section{REFERENCES}

[1] Bušek, D. - Mach, P.

"Properties of Joint Made Using Electrically Conductive Adhesives" Applied Electronics 2005 - International Conference Pilsen. Pilsen: University of West Bohemia, 2005 , p. 49-52. ISBN 80-7043-369-8.

\section{[2] Papež, Václav}

"Non-linearity measurement of passive components by using of intermodulation distortion."

In: International scientific-technical conference XII. DIDMATTECH'99, p.130-133, Nitra 1999

[4] Blackwell, G.R.

"The Electronic Packaging Handbook", New York: CRC Press/IEEE 2000, Conductive adhesives for surface mounting - p.346-369 